\title{
Low-Grade Endometrial Stromal Sarcoma - a Review
}

\author{
Falk C. Thiel ${ }^{a}$ Sonja Halmen ${ }^{b}$ \\ ${ }^{a}$ Department of Gynaecology and Obstetrics, Alb Fils Kliniken, Klinik am Eichert, Göppingen, Germany; \\ ${ }^{b}$ Department of Gynaecology and Obstetrics, Erlangen University Hospital, Erlangen, Germany
}

\section{Keywords}

Uterine sarcoma - Endometrial stromal sarcoma . Undifferentiated endometrial sarcoma

\section{Abstract}

Like other uterine sarcomas, low-grade endometrial stromal sarcomas (LG-ESS) are a very rare tumor entity. In the past, research studies therefore discussed the various different types of the disease in combination. In addition, the classification of endometrial stromal tumors presented difficulties for quite some time so that in earlier studies it was not always possible to precisely distinguish between LG-ESS, high-grade endometrial stromal sarcoma, and undifferentiated uterine sarcoma. For LGESS, surgery with hysterectomy and adnexectomy is the first-line treatment. The benefits of Iymphadenectomy and tumor debulking are unclear. Endocrine therapy with gestagens and aromatase inhibitors is under discussion to provide adjuvant treatment for patients with advanced stages of the disease. As radiotherapy only provides locoregional control, and in view of the usually good prognosis of patients with LG-ESS, its benefits need to be weighed against its side effects. In the case of recurrence, repeat surgery is the first choice. Further research studies viewing LG-ESS as a distinct entity are needed in order to improve treatment options for patients with LG-ESS.

\section{Introduction}

Low-grade endometrial stromal sarcomas (LG-ESS) are a type of uterine sarcoma. Uterine sarcomas are a heterogeneous group of rare tumors of the uterine musculature and uterine connective tissue, and in accordance with the current World Health Organization (WHO) classification, they are distinguished from malignant mesenchymal tumors and malignant mixed epithelial-mesenchymal tumors and classified into the following entities [1, 2]: leiomyosarcoma, LG-ESS, high-grade endometrial stromal sarcoma (HG-ESS), undifferentiated uterine sarcoma (UUS), adenosarcoma, rhabdomyosarcoma, and malignant-type perivascular epithelioid cell tumor.

There are also extremely rare forms such as angiosarcomas, neurogenic sarcomas, osteosarcomas, chondrosarcomas, liposarcomas, myxofibrosarcomas, alveolar soft tissue sarcomas, epithelioid sarcomas, and primitive neuroectodermal tumors. In the WHO classification, these are assigned to the soft tissue sarcomas [3]. Uterine carcinosarcomas (also known as malignant mixed Müllerian tumors) were also formerly included in the group of uterine sarcomas. Nowadays, however, they are assigned to the group of uterine carcinomas [4].

The diagnosis and treatment of uterine sarcomas has recently been reviewed in the German guideline Sarcoma of the Uterus. Guideline of the DGGG (S2k-Level, AWMF Registry No. 015/074, August 2015) [4].

Endometrial stromal sarcomas (ESS) represent only around $0.2 \%$ of all uterine malignancies, but they make up approximately $7-25 \%$ of uterine sarcomas [5-8]. The annual incidence is 0.19 per 100,000 women, and a gradual increase has been observed in the past [9]. ESS is considered to be the second most frequent type of uterine mesenchymal neoplasia after uterine leiomyosarcoma.

In the current WHO classification published in 2014, LG-ESS are classified as endometrial stromal tumors, along with benign en-

\section{KARGER \\ Fax +497614520714

๑) 2018 S. Karger GmbH, Freiburg
Falk Clemens Thiel, MD 
Table 1. FIGO/TNM staging of uterine leiomyosarcomas and endometrial stromal sarcomas

\begin{tabular}{|c|c|c|}
\hline FIGO stage & TNM stage & Definition \\
\hline \multirow[t]{3}{*}{$\mathrm{I} / \mathrm{T} 1$} & & tumor limited to the uterus \\
\hline & IA/Tla & $\leq 5 \mathrm{~cm}$ at its largest diameter \\
\hline & $\mathrm{IB} / \mathrm{T} 1 \mathrm{~b}$ & $>5 \mathrm{~cm}$ at its largest diameter \\
\hline \multirow[t]{3}{*}{$\mathrm{II} / \mathrm{T} 2$} & & tumor extends beyond the uterus to the pelvis \\
\hline & IIA/T2a & $\begin{array}{l}\text { involvement of the adnexa of the uterus (unilateral } \\
\text { or bilateral) }\end{array}$ \\
\hline & $\mathrm{IIB} / \mathrm{T} 2 \mathrm{~b}$ & $\begin{array}{l}\text { tumor spread to extrauterine pelvic tissue excluding } \\
\text { the adnexa }\end{array}$ \\
\hline \multirow[t]{4}{*}{ III/T3 } & & tumor has infiltrated abdominal tissues \\
\hline & IIIA/T3a & 1 site \\
\hline & IIIB/T3b & more than 1 site \\
\hline & IIIC & $\begin{array}{l}\text { metastasis to the pelvic and/or para-aortic lymph } \\
\text { nodes }\end{array}$ \\
\hline IV/T4 & $\begin{array}{l}\text { IVA/T4 } \\
\text { IVB }\end{array}$ & $\begin{array}{l}\text { tumor has infiltrated the bladder and/or rectum } \\
\text { distant metastasis }\end{array}$ \\
\hline
\end{tabular}

dometrial stromal nodules (ESN), HG-ESS, and UUS. ESS are staged along with uterine leiomyosarcomas in accordance with the FIGO and TNM classifications (table 1) $[2,10]$.

Tumor stage is the most important prognostic factor in LG-ESS [11], followed by the patient's age. Another unfavorable factor that has been discussed is uterine morcellation [12-14]. The relevance of mitotic rate, evidence of $\mathrm{p} 53$, and tumor necrosis is unclear $[7,11,15,16]$.

In addition to arising in the uterus, LG-ESS can also develop in extrauterine locations such as the ovaries, the pelvis, or the abdominal cavity, and also the vagina or vulva [17]. Endometriosis is found in $50 \%$ of these cases, giving rise to the suspicion that stromal sarcomas can develop out of endometriosis [18].

Approximately $65 \%$ of the patients are FIGO stage I-II at the time of diagnosis, and around 35\% have FIGO stage III-IV [19]. In patients with tumor stage I-II, the 5-year survival rate is over $90 \%$, while with stages III-IV it is around 50\%.

\section{Classification of Endometrial Stromal Tumors}

The classification of endometrial stromal tumors has been extremely difficult for many decades. These tumors were initially differentiated mainly on the basis of mitoses per 10 high-powered fields (HPF). A mitotic index of $<10 / 10 \mathrm{HPF}$ was associated with a $100 \% 5$-year survival rate, whereas with a mitotic index of $>10 / 10$ HPF the figure was only 55\% [20]. However, it has been known since as early as 1982 that the number of mitoses does not correlate with prognostic validity, making it unsatisfactory and impracticable as a distinguishing criterion. This type of classification is therefore regarded in the literature as obsolete [18, 21]. In 1990, Chang et al. [22] drew a distinction between ESS grade 1, 2, or 3 and undifferentiated sarcomas. Kurihara et al. [23] proposed a distinction between LG-ESS, undifferentiated endometrial sarcomas with nuclear uniformity (UES-U), and undifferentiated endometrial sarco- mas with nuclear pleomorphism (UES-P). In 2012, Lee et al. [2427] achieved a breakthrough by demonstrating a genomic rearrangement that led to a fusion of YWHAE and FAM 22A/B through a translocation of chromosomes 10 and $17(\mathrm{t}(10 ; 17))$. This $14-3-3$ oncoprotein appears to be highly specific for HG-ESS. It was hoped that this genetic feature would make it possible to distinguish between LG-ESS and HG-ESS. The WHO, having in the meantime abandoned the term 'high-grade stromal sarcoma', thus reintroduced the category of HG-ESS in the 2014 classification. Currently, a distinction is now made between LG-ESS, HG-ESS, and UUS. ESS and UUS are distinguished on the basis of morphologic, immunohistochemical, and molecular-pathologic criteria [4].

\section{Pathology}

The cut surface of LG-ESS is yellowish to yellowish-brown, and may also be partly pinkish [18]. LG-ESS can grow intramurally or submucosally, with unclear margins relative to the surrounding tissue [27]. Polypoid growth may lead to displacement of the uterine cavity when the findings are extensive [18].

As malignant tumors, LG-ESS arise from mesenchymal cells that resemble the endometrial stroma in the proliferation phase [2]. Evidence of intratumoral hemorrhage and/or necrosis varies. Typical findings consist of numerous small, uniform cells with linguiform infiltration into the myometrium and into blood and lymph vessels $[18,27]$. The number of mitoses varies but is usually in the low range $(<5 / 10 \mathrm{HPF})$, although larger numbers do not exclude the diagnosis $[18,27]$. It may be difficult to distinguish between LG-ESS with focal glandular differentiation on the one hand and endometriosis on the other [28]. They can be distinguished from ESN by examining their mitotic activity, which is usually lower in ESN $[4,29]$. Infiltration of the myometrium is not observed with ESN, but finger-like projections into the neighboring myometrium are accepted if there are fewer than 3 and they are smaller than $3 \mathrm{~mm}$ [27].

Additional immunohistochemical examinations and molecularpathologic analyses may make it easier to establish a diagnosis. Majority expression of CD10 and WT1 has been reported [4, 30]. Reich et al. [31] noted high levels of expression of estrogen and progesterone receptors in 71 and $95 \%$ of cases, respectively. Most ESS express aromatases [32]. Their expression is associated with tumor growth and may also be relevant to the high recurrence rate [32]. The tumors also sometimes express gonadotrophin-releasing hormone $(\mathrm{GnRH})$ receptors [18]. One therapeutic approach that may be considered is therefore treatment with gestagens, aromatase inhibitors, and $\mathrm{GnRH}$ analogues. Around $45 \%$ of the tumors express androgen receptors [33]. Evidence of smooth-muscle actin, $\beta$-catenin, and pancytokeratins is also possible, whereas CD117 is negative [4]. Cyclin D1 shows variable and heterogeneous nuclear expression in $<10 \%$ of the tumor cells and may be important for distinguishing between these lesions and HG-ESS $[1,4]$.

LG-ESS are a group of lesions with heterogeneous molecularpathologic findings, but several genetic changes observed in them
688
Thiel/Halmen 
are important, such as the translocation $\mathrm{t}(7 ; 17)(\mathrm{p} 15 ; \mathrm{q} 21)$ with fusion of JAZFI-SUZ12 and the fusion genes JAZF1-PHF1 and more rarely EPC1-PHF1, MEAF6-PHF1, ZC3H7-BCOR, and MBTDCXorf67 [27]. A p53 mutation may be found in about one-quarter of ESS; research has shown that changes in p53 may play an important role in the carcinogenesis in these tumors, although they have no influence on prognosis [34].

\section{Clinical Presentation}

ESS typically develop in premenopausal and perimenopausal women with a mean age of 46 (range 18-83 years) [22]. Rare cases have been reported of the tumor developing in connection with tamoxifen or estrogen administration, as well as after radiotherapy $[35,36]$. Obesity, diabetes mellitus, and early menarche are reported to be associated with an increased risk of LG-ESS [17].

These tumors often become apparent through pathologic vaginal bleeding, sometimes also combined with uterine enlargement and associated symptoms such as lower abdominal pain $[2,22]$.

\section{Diagnosis}

In contrast to carcinomas of the endometrium, a diagnosis of LG-ESS as a mesenchymal tumor cannot be securely established using hysteroscopy and fractional curettage. In addition, a clear distinction from benign ESN can only be reliably made after histological analysis of the tumor's entire interface with the neighboring myometrium [17]. Imaging procedures such as ultrasound, computed tomography, and magnetic resonance imaging are not able to display any specific characteristics of LG-ESS [37].

\section{Surgical Treatment}

The primary treatment for LG-ESS is surgery with total hysterectomy (without morcellation) and bilateral salpingo-oophorectomy [4]. Cytoreduction is recommended in advanced tumors with extrauterine manifestations [38, 39]. However, Leath et al. [40] do not regard this as offering any survival advantage in patients with LG-ESS, and in these cases the extent of surgery has to be decided on an individual basis, depending on symptoms and with palliative intent.

It has been shown that LG-ESS are hormone-dependent [12]. It is not clear whether the ovaries can be preserved in young, premenopausal women. Several studies have found a significantly increased rate of recurrence when the ovaries were preserved in premenopausal women [41-43]. In addition, LG-ESS have high levels of steroid receptors and metastasize most frequently from the uterus to the ovaries. A Surveillance Epidemiology and End Results (SEER) analysis did not show any negative effects on overall survival when the ovaries were preserved in premenopausal patients [44]. The decision whether or not to preserve the ovaries therefore always needs to be critically discussed with the patient, with the potential advantage of preserving the ovaries being carefully weighed against the increased risk of recurrence. In view of the tumor biology of LG-ESS, estrogen therapy after bilateral oophorectomy cannot be recommended [45].

Involvement of the pelvic and para-aortic lymph nodes does not appear to have any influence on prognosis. In the study by Chan et al. [19], lymphadenectomy was not associated with any significant improvement in survival, but almost $10 \%$ of the patients who underwent lymphadenectomy had lymph node metastases. Other authors have taken the view that lymphadenectomy may potentially reduce the number of recurrences in the pelvis $[46,47]$, but intraabdominal spread and distant metastases predominate over isolated pelvic recurrences [18]. The SEER data also did not show any benefit of lymphadenectomy in relation to overall survival [44]. Pelvic and para-aortic lymphadenectomy is therefore not a recommended standard procedure in patients with LG-ESS.

\section{Adjuvant Systemic Therapy}

The expression of steroid receptors and aromatases in LG-ESS suggests that adjuvant therapy with gestagens, GnRH analogues, or aromatase inhibitors should be effective. However, a benefit with these endocrine treatments has not been confirmed beyond doubt $[45,46,48]$. In general, the data is limited to case series with small numbers of patients. The heterogeneous distribution pattern of the tumors and potential interaction with androgen receptors are thought to be responsible for the absence of response to gestagen and GnRH analogues, in spite of positive estrogen and progesterone receptors [33]. Gadducci et al. [39] argue in favor of a 24month course of adjuvant gestagen therapy with megestrol. Adjuvant therapy with aromatase inhibitors for 5 years is recommended by some authors [48]. The role of oophorectomy as an adjuvant ablative form of hormonal therapy remains unclear. For tumors that are not removed with healthy margins, adjuvant endocrine therapy is possibly indicated $[39,49,50]$. The response rates are very high, at $82 \%$, and remission periods of more than 10 years have been reported several times [49]. In another study, the efficacy of aromatase inhibitors and gestagens was compared in the adjuvant hormonal treatment of LG-ESS. In patients who were in stage I, gestagen therapy led to a recurrence-free survival (RFS) of 306.2 months (95\% confidence interval (CI) 259.7-352.6 months) compared to 153.1 months (95\% CI 56.8-124.9 months) with aromatase inhibitor therapy and 90.8 months (95\% CI 56.8-124.9 months) without adjuvant endocrine therapy. However, due to severe side effects such as hot flushes, depression, weight gain, and water retention, gestagen treatment was prematurely stopped much more frequently [51].

Starting from FIGO stage III, adjuvant endocrine therapy in accordance with today's standard may be considered but should not be carried out as a general rule [4]. Medroxyprogesterone acetate or megestrol acetate or the aromatase inhibitors letrozole, anastrozole, or exemestane may be used [4].

There are no valid data to show that adjuvant chemotherapy leads to any improvement in survival in patients with LG-ESS. In a 
large observational study conducted by the National Cancer Database, patients with FIGO I ESS received adjuvant chemotherapy. Among 2,414 patients with LG-ESS, 115 (4.8\%) received chemotherapy. A total of 444 (33.4\%) of 1,383 patients with HG-ESS also received adjuvant chemotherapy, and as many as $75.9 \%$ of them (337/444) received multi-agent chemotherapy. A longer survival period was only observed in association with chemotherapy in the group of patients with HG-ESS [52].

\section{Adjuvant Radiotherapy}

In a large epidemiological study conducted in the United States including 3,650 patients with uterine sarcomas, a significant benefit of adjuvant radiotherapy to the pelvis (with or without brachytherapy) was observed in relation to locoregional RFS. The benefit was seen not only for the overall group, but also for the subgroup of patients with ESS ( $\mathrm{n}=312)$, with a 5-year locoregional RFS of 97 versus $93 \%$ and an 8-year locoregional RFS of 97 versus $87 \%$ [53]. However, another large study including 1,010 patients with ESS did not find that adjuvant pelvic radiotherapy had any positive effect on overall survival [54]. A randomized phase III study including a total of 224 patients with uterine sarcomas also included 28 patients with ESS. The patients were randomly assigned to an arm that received postoperative adjuvant pelvic radiotherapy ( $51 \mathrm{~Gy}$ in 28 fractions over 5 weeks), in comparison with observation alone [55]. Although there was a reduction in the local recurrence rate ( $\mathrm{n}=14$ vs. $24 ; \mathrm{p}=0.004$ ), no effect on progression-free survival or overall survival was seen. The study did not conduct a subgroup analysis of patients with ESS.

Postoperative radiotherapy in patients with ESS thus only appears to improve locoregional control, so that the medium-term and long-term side effects of pelvic irradiation need to be weighed carefully against what is in any case a good prognosis in relation to locoregional recurrences [4].

\section{Recurrences and Distant Metastases}

The risk of recurrence in LG-ESS is $10-20 \%$, and late recurrences after more than 10-30 years are characteristic of the disease $[25,26]$. Recurrences may appear locally in the vagina or lesser pelvis, or as distant metastases in the abdominal wall or lung [56]. Only $40 \%$ of recurrences are limited exclusively to the lesser pelvis; $60 \%$ of them occur intra-abdominally, as distant metastases, or as a combination of all forms of dissemination [57]. The median period to the appearance of recurrences is 5.4-9.3 years in stages I and II and only 9 months in stages III and IV (FIGO prior to 2009) [57, 58]. Distant metastases and recurrences do not show any association with positive lymph nodes. Even in patients with negative lymph node status, recurrence rates of up to $30 \%$ within 2 years are observed [59].

Surgery, radiotherapy, and systemic therapy are regarded as potential treatment options in patients with recurrences of and metastases from uterine sarcomas [60], but only complete surgical resection is associated with an increased rate of cure and prolonged survival [61-64]. After each recurrence or isolated metastasis, the extent to which complete resection is possible should therefore be checked. Due to the slow growth of the lesions, ESS can also be repeatedly successfully resected [37].

Percutaneous radiotherapy can be used for palliative treatment in patients with local or locoregional recurrences of uterine sarcomas $[65,66]$. However, even in combination with chemotherapy, tumor progression occurs in more than $50 \%$ of the patients within 2 years [65-67]. Intraoperative radiotherapy or percutaneous irradiation can achieve a 3-year survival rate of $53 \%$ and a median survival period of 18 months after surgical treatment in patients with residual tumors [54].

Due to the high level of expression of estrogen and progesterone receptors in LG-ESS, gestagens or aromatase inhibitors can be administered in patients with postoperative residual tumor, inoperable recurrences, or distant metastases [49, 68-70].

Chemotherapy should only be used when other options have been exhausted. There are no studies showing any superiority of combination therapy over monotherapy. The data available are based only on case reports and phase II studies in which other types of uterine sarcoma were also treated [71].

Due to the expression of epidermal growth factor receptor in $70 \%$ of ESS, treatment with monoclonal antibodies or tyrosine kinase inhibitors is conceivable $[18,72]$. There have been individual observations of complete remission during treatment with imatinib [73].

\section{Conclusion}

Like the other uterine sarcomas, LG-ESS are a very rare tumor entity. The various diseases used to therefore be combined together in past research studies. In addition, the classification of endometrial stromal tumors presented difficulties for a considerable period so that earlier studies were not always able to distinguish precisely between LG-ESS, HG-ESS, and UUS. For LG-ESS, surgery with hysterectomy and adnexectomy is the first-line treatment. The benefits of lymphadenectomy or tumor debulking are unclear. Endocrine therapies with gestagens and aromatase inhibitors are under discussion as adjuvant treatments in patients with advanced stages. Since it only provides locoregional control in patients who usually have a good prognosis with LG-ESS, radiotherapy needs to be carefully weighed up in relation to its side effects. Repeat surgery is also the treatment of choice in cases of recurrence.

In order to improve the treatment options available for LG-ESS, further research studies need to be conducted in which LG-ESS is considered as a distinct entity.

\section{Disclosure Statement}

F.T. and S.H. hereby declare that they do not have any potential conflicts of interest in relation to the publication of this article. F.T. is a member of the guideline group in Germany on 'uterine sarcomas', as a representative of the German Society for Gynaecology and Obstetrics (Deutsche Gesellschaft für Gynäkologie und Geburtshilfe e.V., DGGG).
Thiel/Halmen 


\section{References}

1 Conklin CM, Longacre TA: Endometrial stromal tumors: the new WHO classification. Adv Anat Pathol 2014;21:383-393.

2 Oliva E, Carcangiu ML, Carinelli SG, et al.: Mesenchymal tumors of the uterus; in Kurman RJ, Carcangiu ML, Herrington CS, Young RH (eds): WHO Classification of Tumours of Female Reproductive Organs. Lyon, IARC Press, 2014, pp. 135-147.

3 Fletcher CDM, Bridge JA, Hogendoorn PCW, Mertens F: WHO Classification of Tumours of Soft Tissue and Bone. Lyon, IARC Press, 2013.

$\checkmark 4$ Denschlag D, Thiel FC, Ackermann S, et al.: Sarcoma of the uterus. Guideline of the DGGG (S2k-level, AWMF registry no. 015/074, August 2015). Geburtshilfe Frauenheilkd 2015;75:1028-1042.

5 Hosh M, Antar S, Nazzal A, et al.: Uterine sarcoma: analysis of 13,089 cases based on surveillance, epidemiology, and end results database. Int J Gynecol Cancer 2016;26:1098-1104.

6 Brooks SE, Zhan M, Cote T, Baquet CR: Surveillance, epidemiology, and end results analysis of 2677 cases of uterine sarcoma 1989-1999. Gynecol Oncol 2004;93: 204-208.

7 Abeler VM, Røyne O, Thoresen S, et al.: Uterine sarcomas in Norway. A histopathological and prognostic survey of a total population from 1970 to 2000 including 419 patients. Histopathology 2009;54:355-364.

8 Nordal RR, Kristensen GB, Stenwig AE, et al.: An evaluation of prognostic factors in uterine carcinosarcoma. Gynecol Oncol 1997;67:316-321.

9 Harlow BL, Weiss NS, Lofton S: The epidemiology of sarcomas of the uterus. J Natl Cancer Inst 1986;76: 399-402.

10 Wittekind C, Meyer HJ: TNM-Klassifikation Maligner Tumoren. Weinheim, Wiley-VHC Verlag, 2010

11 Chew I, Oliva E: Endometrial stromal sarcomas: a review of potential prognostic factors. Adv Anat Pathol 2010;17:113-121.

12 Einstein MH, Barakat RR, Chi DS, et al.: Management of uterine malignancy found incidentally after supracervical hysterectomy or uterine morcellation for presumed benign disease. Int J Gynecol Cancer 2008; 18: 1065-1070.

13 Della Badia C, Karini H: Endometrial stromal sarcoma diagnosed after uterine morcellation in laparoscopic supracervical hysterectomy. J Minim Invasive Gynecol 2010;17:791-793.

14 Park JY, Park SK, Kim DY, et al.: The impact of tumor morcellation during surgery on the prognosis of patients with apparently early uterine leiomyosarcoma. Gynecol Oncol 2011;122:255-259.

15 Trope CG, Abeler VM, Kristensen GB: Diagnosis and treatment of sarcoma of the uterus. A review. Acta Oncol 2012;51:694-705.

16 Park JY, Kim KR, Nam JH: Immunohistochemical analysis for therapeutic targets and prognostic markers in low-grade endometrial stromal sarcoma. Int J Gynecol Cancer 2013;23:81-89.

17 Hoang L, Chiang S, Lee CH: Endometrial stromal sarcomas and related neoplasms: new developments and diagnostic considerations. Pathology 2018;50:162-177.

18 Köhler G, Evert M: Uterine Sarkome und Mischtumoren: Handbuch und Bildatlas zur Diagnostik und Therapie. Berlin, New York, NY, de Gruyter, 2009

19 Chan JK, Kawar NM, Shin JY, et al.: Endometrial stromal sarcoma: a population-based analysis. Br J Cancer 2008;99:1210-1215.

20 Norris HJ, Taylor HB: Mesenchymal tumors of the uterus. I. A clinical and pathological study of 53 endometrial stromal tumors. Cancer 1966;19:755-766.
Evans HL: Endometrial stromal sarcoma and poorly differentiated endometrial sarcoma. Cancer 1982;50: 2170-2182.

22 Chang KL, Crabtree GS, Lim-Tan SK, Kempson RL, Hendrickson MR: Primary uterine endometrial stromal neoplasms. A clinicopathologic study of 117 cases. Am J Surg Pathol 1990;14:415-438.

23 Kurihara S, Oda Y, Ohishi Y, et al.: Endometrial stromal sarcomas and related high-grade sarcomas: immunohistochemical and molecular genetic study of 31 cases. Am J Surg Pathol 2008;32:1228-1238.

24 Lee CH, Ali RH, Rouzbahman M, et al.: Cyclin D1 as a diagnostic immunomarker for endometrial stromal sarcoma with YWHAE-FAM22 rearrangement. Am J Surg Pathol 2012;36:1562-1570.

25 Lee CH, Marino-Enriquez A, Ou W, et al.: The clinicopathologic features of YWHAE-FAM22 endometrial stromal sarcomas: a histologically high-grade and clinically aggressive tumor. Am J Surg Pathol 2012;36: 641-653.

26 Lee CH, Ou WB, Marino-Enriquez A, et al.: 14-3-3 fusion oncogenes in high-grade endometrial stromal sarcoma. Proc Natl Acad Sci U S A 2012;109:929-934.

27 Ali RH, Rouzbahman M: Endometrial stromal tumours revisited: an update based on the 2014 WHO classification. J Clin Pathol 2015;68:325-332.

28 McCluggage WG, Ganesan R, Herrington CS: Endometrial stromal sarcomas with extensive endometrioid glandular differentiation: report of a series with emphasis on the potential for misdiagnosis and discussion of the differential diagnosis. Histopathology 2009;54: 365-373.

29 Wells M, Oliva E, Palacios J, Prat J: Mixed epithelial and mesenchymal tumors of the uterus; in Kurman RJ, Carcangiu ML, Herrington CS, Young RH (eds): WHO Classification of Tumours of Female Reproductive Organs. Lyon, IARC Press, 2014, vol 6, pp. 135147.

30 Oliva E, Clement PB, Young RH: Epithelioid endometrial and endometrioid stromal tumors: a report of four cases emphasizing their distinction from epithelioid smooth muscle tumors and other oxyphilic uterine and extrauterine tumors. Int J Gynecol Pathol 2002;21:48-55.

31 Reich O, Regauer S, Urdl W, Lahousen M, Winter R: Expression of oestrogen and progesterone receptors in low-grade endometrial stromal sarcomas. $\mathrm{Br} \mathrm{J}$ Cancer 2000;82:1030-1034.

32 Reich O, Regauer S: Aromatase expression in lowgrade endometrial stromal sarcomas: an immunohistochemical study. Mod Pathol 2004;17:104-108.

33 Moinfar F, Regitnig P, Tabrizi AD, Denk H, Tavassoli FA: Expression of androgen receptors in benign and malignant endometrial stromal neoplasms. Virchows Arch 2004;444:410-414.

34 Nordal RR, Kristensen GB, Stenwig AE, Trope CG, Nesland JM: Immunohistochemical analysis of p53 protein in uterine sarcomas. Gynecol Oncol 1998;70: 45-48.

35 Beer TW, Buchanan R, Buckley CH: Uterine stromal sarcoma following tamoxifen treatment. J Clin Pathol 1995;48:596.

36 Meredith RF, Eisert DR, Kaka Z, et al.: An excess of uterine sarcomas after pelvic irradiation. Cancer 1986; 58:2003-2007.

37 Amant F, Coosemans A, Debiec-Rychter M, Timmerman D, Vergote I: Clinical management of uterine sarcomas. Lancet Oncol 2009;10:1188-1198.

38 Gadducci A, Sartori E, Landoni F, et al.: Endometrial stromal sarcoma: analysis of treatment failures and survival. Gynecol Oncol 1996;63:247-253.
Gadducci A, Cosio S, Romanini A, Genazzani AR: The management of patients with uterine sarcoma: a debated clinical challenge. Crit Rev Oncol Hematol 2008; 65:129-142.

40 Leath CA 3rd, Huh WK, Hyde J Jr, et al.: A multi-institutional review of outcomes of endometrial stromal sarcoma. Gynecol Oncol 2007;105:630-634.

41 Amant F, De Knijf A, Van Calster B, et al.: Clinica study investigating the role of lymphadenectomy, surgical castration and adjuvant hormonal treatment in endometrial stromal sarcoma. Br J Cancer 2007;97: 1194-1199.

42 Berchuck A, Rubin SC, Hoskins WJ, et al.: Treatment of endometrial stromal tumors. Gynecol Oncol 1990; 36:60-65.

43 Li N, Wu LY, Zhang HT, et al.: Treatment options in stage I endometrial stromal sarcoma: a retrospective analysis of 53 cases. Gynecol Oncol 2008;108:306-311.

44 Shah JP, Bryant CS, Kumar S, et al.: Lymphadenectomy and ovarian preservation in low-grade endometrial stromal sarcoma. Obstet Gynecol 2008;112:11021108.

45 Chu MC, Mor G, Lim C, et al.: Low-grade endometrial stromal sarcoma: hormonal aspects. Gynecol Oncol 2003;90:170-176.

46 Thomas MB, Keeney GL, Podratz KC, Dowdy SC: Endometrial stromal sarcoma: treatment and patterns of recurrence. Int J Gynecol Cancer 2009; 19:253-256.

47 Riopel J, Plante M, Renaud MC, Roy M, Tetu B: Lymph node metastases in low-grade endometrial stromal sarcoma. Gynecol Oncol 2005;96:402-406.

48 Reich O, Singer C, Hudelist G, Regauer S: Estrogen sulfotransferase expression in endometrial stromal sarcomas: an immunohistochemical study. Pathol Res Pract 2007;203:85-87.

49 Dahhan T, Fons G, Buist MR, Ten Kate FJ, van der Velden J: The efficacy of hormonal treatment for residual or recurrent low-grade endometrial stromal sarcoma. A retrospective study. Eur J Obstet Gynecol Reprod Biol 2009; 144:80-84

50 Reed NS: The management of uterine sarcomas. Clin Oncol (R Coll Radiol) 2008;20:470-478.

51 Deshmukh U, Black J, Perez-Irizarry J, et al.: Adjuvant hormonal therapy for low-grade endometrial stromal sarcoma. Reprod Sci 2018;Epub ahead of print.

52 Seagle BL, Sobecki-Rausch J, Strohl AE, et al: Prognosis and treatment of uterine leiomyosarcoma: a national cancer database study. Gynecol Oncol 2017;145: 61-70.

53 Sampath S, Schultheiss TE, Ryu JK, Wong JY: The role of adjuvant radiation in uterine sarcomas. Int J Radiat Oncol Biol Phys 2010;76:728-734.

54 Barney B, Tward JD, Skidmore T, Gaffney DK: Does radiotherapy or lymphadenectomy improve survival in endometrial stromal sarcoma? Int J Gynecol Cancer 2009; 19:1232-1238.

55 Reed NS, Mangioni C, Malmstrom H, et al.; European Organisation for Research and Treatment of Cancer Gynaecological Cancer Group: Phase III randomised study to evaluate the role of adjuvant pelvic radiotherapy in the treatment of uterine sarcomas stages I and II: an European Organisation for Research and Treatment of Cancer Gynaecological Cancer Group study (protocol 55874). Eur J Cancer 2008;44:808-818.

56 Bai H, Yang J, Cao D, et al.: Ovary and uterus-sparing procedures for low-grade endometrial stromal sarcoma: a retrospective study of 153 cases. Gynecol Oncol 2014;132:654-660.

57 Kim WY, Lee JW, Choi CH, et al.: Low-grade endometrial stromal sarcoma: a single center's experience with 22 cases. Int J Gynecol Cancer 2008;18:1084-1089. 
58 Amant F, Moerman P, Cadron I, et al.: The diagnostic problem of endometrial stromal sarcoma: report on six cases. Gynecol Oncol 2003;90:37-43.

59 Goff BA, Rice LW, Fleischhacker D, et al.: Uterine leiomyosarcoma and endometrial stromal sarcoma: lymph node metastases and sites of recurrence. Gynecol Oncol 1993;50:105-109.

60 Nam JH: Surgical treatment of uterine sarcoma. Best Pract Res Clin Obstet Gynaecol 2011;25:751-760.

61 Levenback C, Rubin SC, McCormack PM, et al.: Resection of pulmonary metastases from uterine sarcomas. Gynecol Oncol 1992;45:202-205.

62 Weiser MR, Downey RJ, Leung DH, Brennan MF: Repeat resection of pulmonary metastases in patients with soft-tissue sarcoma. J Am Coll Surg 2000;191: 184-190; discussion 190-181.

63 Leitao MM, Brennan MF, Hensley M, et al.: Surgical resection of pulmonary and extrapulmonary recurrences of uterine leiomyosarcoma. Gynecol Oncol 2002;87:287-294.
64 Bernstein-Molho R, Grisaro D, Soyfer V, Safra T, Merimsky O: Metastatic uterine leiomyosarcomas: a single-institution experience. Int J Gynecol Cancer 2010;20:255-260.

65 Weitmann HD, Knocke TH, Kucera H, Potter R: Radiation therapy in the treatment of endometrial stromal sarcoma. Int J Radiat Oncol Biol Phys 2001;49: 739-748.

66 Dusenbery KE, Potish RA, Judson P: Limitations of adjuvant radiotherapy for uterine sarcomas spread beyond the uterus. Gynecol Oncol 2004;94:191-196.

67 Kortmann B, Reimer T, Gerber B, Klautke G, Fietkau $\mathrm{R}$ : Concurrent radiochemotherapy of locally recurrent or advanced sarcomas of the uterus. Strahlenther Onkol 2006;182:318-324.

68 Cheng X, Yang G, Schmeler KM, et al.: Recurrence patterns and prognosis of endometrial stromal sarcoma and the potential of tyrosine kinase-inhibiting therapy. Gynecol Oncol 2011;121:323-327.
69 Pink D, Lindner T, Mrozek A, et al.: Harm or benefit of hormonal treatment in metastatic low-grade endometrial stromal sarcoma: single center experience with 10 cases and review of the literature. Gynecol Oncol 2006;101:464-469.

70 Maluf FC, Sabbatini P, Schwartz L, Xia J, Aghajanian C: Endometrial stromal sarcoma: objective response to letrozole. Gynecol Oncol 2001;82:384-388.

71 Harter P, Canzler U, Lueck H, et al.: Pegylated liposomal doxorubicin and carboplatin in malignant mixed epithelial mesenchymal and mesenchymal gynecologic tumors: a phase II trial of the AGO study group. J Clin Oncol 2011;29:5093-5093

72 Moinfar F, Azodi M, Tavassoli FA: Uterine sarcomas. Pathology 2007;39:55-71.

73 Kalender ME, Sevinc A, Yilmaz M, Ozsarac C, Camci C: Detection of complete response to imatinib mesylate (Glivec/Gleevec) with 18F-FDG PET/CT for low-grade endometrial stromal sarcoma. Cancer Chemother Pharmacol 2009;63:555-559. 